\title{
Altersgerechtes Webdesign
}

\author{
Alexander Seifert, Alireza Darvishy \& Stephan Roth
}

Webseiten sollten «altersgerecht und für alle nutzbar sein». Dieser Forderung wurde in einem interdisziplinären Forschungsprojekt nachgegangen. Entstanden ist eine hilfreiche Broschüre für die Praxis.

\section{Conception de sites web adaptée à l'âge}

Les sites Web doivent « être adaptés à l'âge et utilisables par tous ». Cette exigence a été explorée dans un projet de recherche interdisciplinaire qui a débouché sur une brochure utile pour la pratique.

DOI 10.1024/2297-5160/a000012

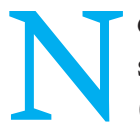
eben fehlenden persönlichen Unterstützungen sind es nach der Studie von Seifert und Schelling (siehe Beitrag in diesem Heft, S. 18) vorwiegend personenbezogene Hindernisse und Ängste, die eine Internetnutzung erschweren. Daneben sind es aber auch die Webseiten selbst, die durch eine zu wenig benutzerfreundliche Gestaltung vermeidbare Hürden aufbauen.

\section{Forschung mit Seniorinnen und Senioren führt zu praktischen Empfehlungen}

Um die Bedürfnisse und Anforderungen der Generation 65 plus an ein benutzerfreundliches Webdesign genauer zu untersuchen, wurden in einem Projekt der Zürcher Hochschule für Angewandte Wissenschaften (zhaw) in Zusammenarbeit mit dem Zentrum für Gerontologie der Univer- sität Zürich ältere Personen zum Thema «altersgerechtes Internet» befragt. Hierzu wurden vier Fokusgruppen mit insgesamt 24 älteren Teilnehmenden (Durchschnittsalter 73 Jahre), die das Internet regelmässig nutzen, gebildet.

Ziel der Fokusgruppen war es, die Sichtweise der älteren Nutzer/-innen sowie auftretende Barrieren aufgrund des Webdesigns zu erheben. Ein wichtiges Ergebnis war, dass die teilnehmenden Personen auf eine gute Gestaltung der Webseiten grossen Wert legen. So berichteten einige, sie hätten bereits Webseiten wegen einer schlechten Gestaltung/Benutzerführung nicht wunschgemäss nutzen können. Im Anschluss an die Gruppeninterviews wurden die Aussagen mit dem gegenwärtigen Stand der Fachliteratur verglichen und innerhalb zweier Expertenrunden diskutiert und ergänzt.

Auf Grundlage dieser mehrstufigen Erhebung durch Literaturrecherche, Anwendergruppen und Expertenrunden konnten Empfehlungen für eine «altersgerechte Webseitengestaltung» formuliert werden. Diese Empfehlungen umfassen u.a. Themenbereiche wie die Übersichtlichkeit von Webseiten, die Lesbarkeit und Wahrnehmbarkeit von Inhalten sowie die Benutzerfreundlichkeit der Navigation.

Die Empfehlungen wurden in einer Broschüre systematisch zusammengetragen. Diese Broschüre und ein E-Learning-Modul können unter www.ageweb.ch abgerufen werden. Für Anfang 2016 ist eine zweite Broschüre zum Thema «Altersgerechte mobile Applikationen» geplant.

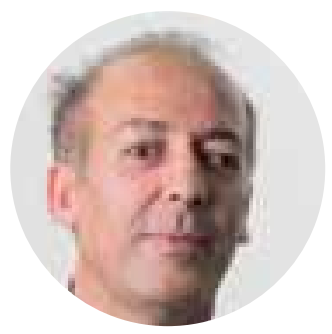

Prof. Dr. Alireza Darvishy ist Dozent und Leiter der Fachstelle ICT-Accessibility an der Zürcher Hochschule für Angewandte Wissenschaften (zhaw).

alireza.darvishy@zhaw.ch 


\section{Gesundheit, Krankheit und das Gesundheits- system verstehen}
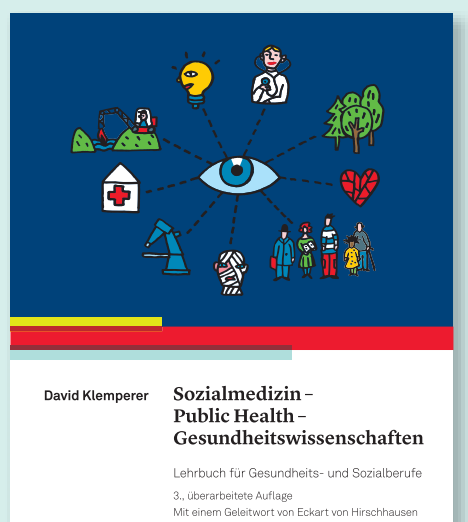

(1) hogrefe

www.hogrefe.com

David Klemperer

\section{Sozialmedizin - Public Health -} Gesundheitswissenschaften

Lehrbuch für Gesundheits- und Sozialberufe

3., überarb. Aufl. 2015. 384 S., 153 Abb., Kt

$€ 29.95$ / CHF 39.90

ISBN 978-3-456-85550-9

AUCH ALS E-BOOK

Dieses Lehrbuch will die Gesundheits- und Sozialberufe darin unterstützen, ihre berufliche Praxis weiterzuentwickeln. Dafür ist eine breite Wissensbasis hilfreich, die dieses Buch praxisnah vermittelt.

Dazu zählt das Wissen über

- Individualmedizin und Bevölke-

rungsgesundheit (Public Health)

- Modelle von Gesundheit und Krankheit

- Epidemiologie und Forschungsme-

thoden

- Prinzipien einer evidenzbasierten beruflichen Praxis
- Theorie und Praxis von Gesundheitsförderung und Krankheitsprävention

- soziale Ungleichheiten der Gesundheit.

Das Buch richtet sich sowohl an Studierende als auch an bereits Berufstätige. Angesprochen sind ebenfalls Patientenvertreter in der Selbstverwaltung, Krankenkassenmitarbeiter, Mitglieder und Mitarbeiter der gesundheitsbezogenen Selbsthilfe, Journalisten und an Gesundheitspolitik Interessierte. 